\title{
NANOSCALE TUNING FORK CAVITY OPTOMECHANICAL TRANSDUCERS WITH DESIGN-ENABLED FREQUENCY TUNING AND TEMPERATURE COMPENSATION
}

\author{
Rui Zhang ${ }^{1}$, Robert Ilic ${ }^{2}$, Yuxiang Liu ${ }^{1}$, and Vladimir Aksyuk ${ }^{2}$ \\ ${ }^{1}$ Department of Mechanical Engineering, Worcester Polytechnic Institute, USA \\ ${ }^{2}$ Center for Nanoscale Science and Technology, National Institute of Standards and Technology, USA
}

\begin{abstract}
In this work, we design, fabricate and characterize monolithic, nanoscale $\mathrm{Si}_{3} \mathrm{~N}_{4}$ tuning fork cavity optomechanical transducers with design enabled tuning of mechanical resonant frequencies and passive temperature compensation. Both frequency tuning and temp erature compensation are achieved by the design of a nonlinear mechanical clamp. The compensation reduces the temperature sensitivity of frequency by $\approx 60$ times, achieving fractional frequency sensitivity of $(3.2 \pm 0.4) \cdot 10^{-6} \mathrm{~K}^{-1}$. The design simultaneously increases the stress in the tuning fork by more than a factor of two relative to the residual stress, and makes the resulting high resonator frequency insensitive to the residual stress and temperature variations. These stable resonators may find uses for stable transduction of force and motion in MEMS devices via frequency readout.
\end{abstract}

\section{INTRODUCTION}

Many mechanical sensors, such as high performance inertial sensors, demand integrated mechanical motion detection with low noise, high bias stability, large dynamic ranges, and high bandwidths. Monitoring the resonant frequency change of a highquality mechanical resonator is a widely accepted approach for motion detection. This approach is immune to low-frequency electronic noise and bias drift, and both a high mechanical frequency $\left(f_{\mathrm{m}}\right)$ and a high mechanical quality factor $\left(Q_{\mathrm{m}}\right)$ are preferred since high $f_{\mathrm{m}}$ can provide high transduction bandwidth/temporal resolution and the force sensitivity scales as $1 /\left(f_{\mathrm{m}}{ }^{0.5} Q_{\mathrm{m}}{ }^{0.5}\right)[1]$. Nanomechanical resonators made of high intrinsic tensile stress silicon nitride $\left(\mathrm{Si}_{3} \mathrm{~N}_{4}\right)$ have shown great potential for such sensing applications [2] because of their high mechanical quality factors, high frequencies, and facile fabrication. Recently developed nanoscale $\mathrm{Si}_{3} \mathrm{~N}_{4}$ tuning fork resonators [3] achieve very large frequency-quality factor $\left(f_{\mathrm{m}} Q_{\mathrm{m}}\right)$ products $\left(Q_{\mathrm{m}} \approx 2.2 \times 10^{5}\right.$ at $f_{\mathrm{m}} \approx$ $29 \mathrm{MHz}$ ) through a design-enabled stress tuning approach, and open unprecedented opportunities for on-chip motion metrology and sensing. However, the frequency of such tuning forks is determined by both designed geometry and the intrinsic stress $\left(\sigma_{0}\right)$, and $\sigma_{0}$ varies with different fabrication processes [4]. In addition, the performance of the tuning fork mechanical resonator can be influenced by ambient environmental factors, especially the temperature [5]. For example, temperature fluctuations can influence the tensile stress in a doubly-clamped $\mathrm{Si}_{3} \mathrm{~N}_{4}$ tuning fork, due to the difference of coefficient of thermal expansion (CTE) between the $\mathrm{Si}_{3} \mathrm{~N}_{4}$ device layer and the $\mathrm{Si}$ substrate. Thermal stress fluctuations in tum influence the thermal stability of the resonant frequency [6]. The majority of approaches made to compensate temperature-induced frequency variation rely on matching different materials [7] or active temperature control [8].

Here we design, fabricate, and characterize a monolithic nonlinear mechanical clamp for single-layer $\mathrm{Si}_{3} \mathrm{~N}_{4}$ tuning fork nanomechanical resonators that renders the natural frequencies insensitive to temperature fluctuations by a passive, design-enabled compensation method. In addition, by introducing a reference length

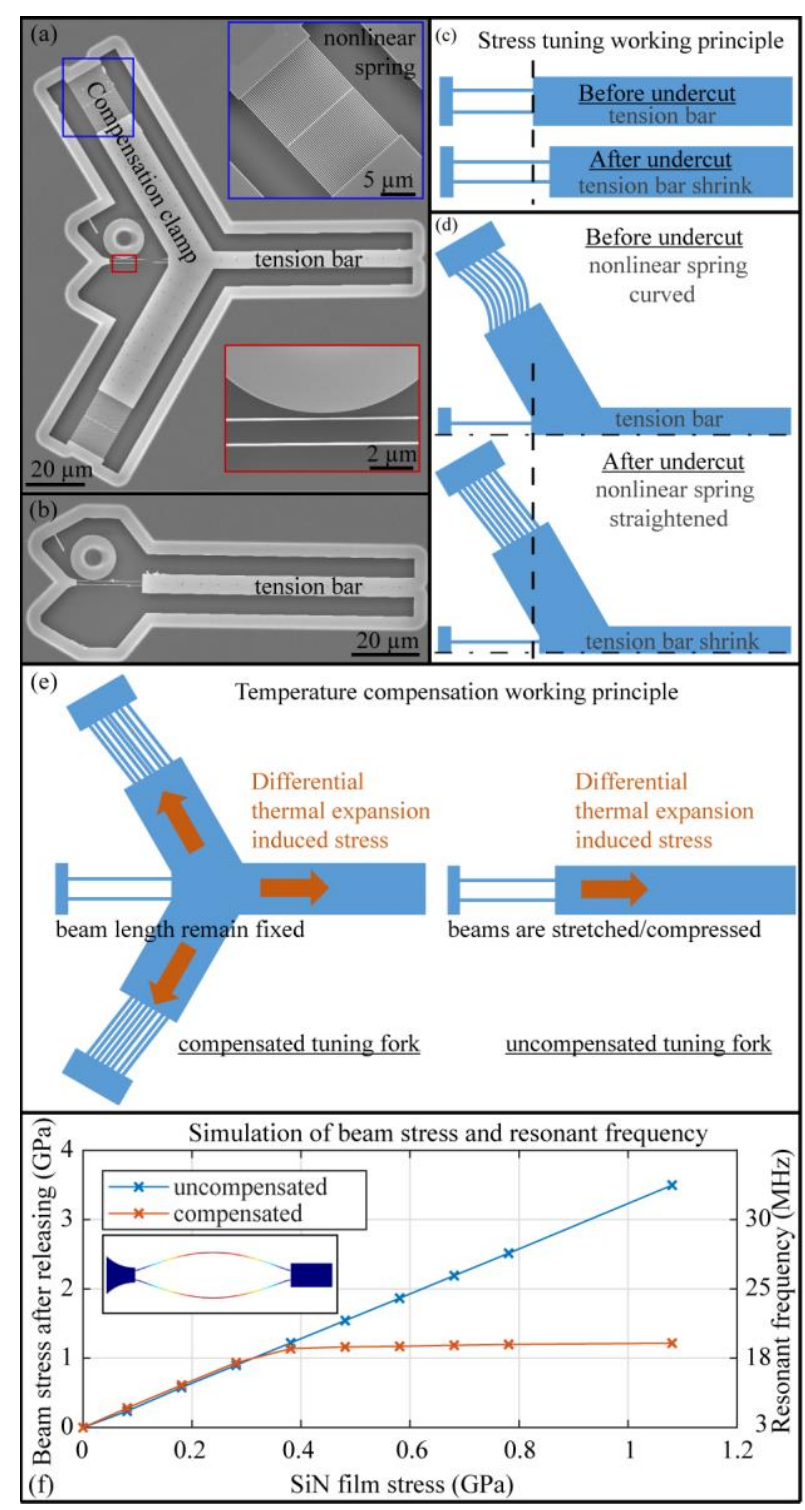

Figure 1: (a) SEM of a tuning fork with temperature compensation and integrated photonic readout. (Top inset) Zoom-in of straightened nonlinear springs. (Bottom inset) Coupling region between tuning fork beam and microdisk optical resonator. (b) SEM of tuning fork without temperature compensation. (c) Working principle of stress tuning in uncompensated tuning fork. (d) Working principle of stress tuning in compensated tuning fork. (e) Working principle of temperature compensation. (f) Simulation of beam stress and device frequency with different $\mathrm{Si}_{3} \mathrm{~N}_{4}$ film stress. The inset shows the simulated tuning fork in-plane squeezing mode shape with exaggerated deformation. 
scale via the device lay out, this clamp design can achieve the same designed resonator strain and resonant frequency over a wide range of intrinsic stress values and enables geometry-determined on-chip stress tuning capability. Different temperature compensation strengths including under-compensation, proper compensation, and over-compensation can be achieved by varying the design and are demonstrated in the experiments. The device with the best temperature compensation has a temperature sensitivity of $-54 \mathrm{~Hz} / \mathrm{K} \pm 6 \mathrm{~Hz} / \mathrm{K}$ with $f_{\mathrm{m}} \approx 16.5 \mathrm{MHz}$. The tuning fork without temperature compensation has a temperature sensitivity of $5360 \mathrm{~Hz} / \mathrm{K} \pm 170 \mathrm{~Hz} / \mathrm{K}$ with $f_{\mathrm{m}} \approx 27.76 \mathrm{MHz}$. All reported statistical uncertainties are $95 \%$ confidence ranges unless noted otherwise. The nonlinear mechanical clamp can be adapted for a wide range of doubly-clamped nanomechanical resonators to reduce temperature sensitivity.

Scanning electron microscopy (SEM) images of temperature compensated and uncompensated tuning forks are shown in Figures 1(a) and (b), respectively. The uncompensated tuning fork consists of two parallel cantilever beams, and is doubly clamped with a long tension bar on the right. By comparison, the compensated tuning fork has two additional inclined clamps, resulting in a Y-shaped clamp at the right end of the tuning fork. Each of the inclined clamps is attached to the supporting structure by an array of nonlinear springs. All the devices are fabricated in a $250 \mathrm{~nm}$ thick stoichiometric $\mathrm{Si}_{3} \mathrm{~N}_{4}$ layer, which is grown by low-pressure chemical vapor deposition (LPCVD) on a $3 \mu \mathrm{m}$ thick silicon dioxide $\left(\mathrm{SiO}_{2}\right)$ layer on a silicon substrate. We first used electron-beam (Ebeam) lithography to define the geometry in a layer of positive tone E-beam resist. The pattern was then transferred to the $\mathrm{Si}_{3} \mathrm{~N}_{4}$ film by a $\mathrm{CHF}_{3} / \mathrm{Ar} / \mathrm{O}_{2}$ inductively-coupled plasma reactive ion etch (ICP RIE). Device fabrication is finished by a buffered oxide etch (BOE) undercut, which selectively removes the $\mathrm{SiO}_{2}$ to release the device with minimal etching of $\mathrm{Si}_{3} \mathrm{~N}_{4}$.

The nominal length and width of the tuning fork beams are $20 \mu \mathrm{m}$ and $150 \mathrm{~nm}$, respectively, in all the fabricated devices. The tension bar on the right-hand side of the tuning fork is $80 \mu \mathrm{m}$ long. In the uncompensated device, the tension bar shrinks after device release due to the redistribution of the initially uniform $\mathrm{Si}_{3} \mathrm{~N}_{4}$ stress, such that the stress in the tuning fork beam increases, as shown in Figure 1(c). Because the length of the tension bar determines the final stress in the tuning fork after undercut, one can tune the fork mechanical frequency simply by the design of the tension bar. This design-enabled tuning has been demonstrated in our previous work to achieve an up-to-three-fold stress increase, compared with the intrinsic $\mathrm{Si}_{3} \mathrm{~N}_{4}$ film stress, and 1.5 times increase in the fork fundamental frequency compared with the fork without the tension bar [3].

In the temp erature-compensated device (shown in Figure 1(a)), the Y-shaped clamp includes two symmetric inclined clamps and a tension bar. Each of the two symmetric, inclined compensation clamps has an array of nonlinear springs close to one end. The springs are curved before undercut with the shape defined by one period of a cosine function $(0.48 \mu \mathrm{m}) \times \cos (\mathrm{x} /(20 \mu \mathrm{m}) \times 2 \pi)$, where $0.48 \mu \mathrm{m}$ is the modulation of the cosine geometry and $\mathrm{x}$ is the position in micrometers along the longitudinal direction. The symmetric inclined clamps and the curved springs allow the tuning fork beam stress to be insensitive to the intrinsic $\mathrm{Si}_{3} \mathrm{~N}_{4}$ stress, while retaining the stress tuning cap ability, as described below. During the release process (Figure 1(d)), the tension bar first shrinks, as in the uncompensated device, stretching the tuning forks and straightening the curved springs in the inclined clamps. As a result, the tuning fork beam stress and frequency are tuned by the tension bar design, until the curved springs are fully straightened. If the intrinsic $\mathrm{Si}_{3} \mathrm{~N}_{4}$ film stress is large enough to fully straighten the nonlinear springs, the stiffness of the straight nonlinear springs increases significantly compared with that of curved springs. This is because the spring deformations change from curved beam bending to straight beam stretching. The inclined clamps then provide an effective balancing force in the opposite direction of the stretching force produced by the tension bar. As a result, the right-hand end-point of the tuning fork beams is fixed and hence the tuning fork beam stress as well as mechanical frequency become insensitive to the intrinsic $\mathrm{Si}_{3} \mathrm{~N}_{4}$ film stress.

The stress insensitivity of the frequency enabled by the clamp design is verified by a proof-of-concept Finite-Element-Method (FEM) simulation. The simulated final beam stress and mechanical frequency at different $\mathrm{Si}_{3} \mathrm{~N}_{4}$ intrinsic stress are shown in Figure 1(f). In the uncompensated device, the beam stress is linearly proportional to the intrinsic stress. In the compensated device, the beam stress in the tuning fork increases similarly for small intrinsic stress, before the nonlinear springs are fully straightened. However, the beam stress is almost constant for the intrinsic stress above a threshold value at which the nonlinear springs become straight and stiff. The final beam stress in the compensated device is determined by the clamp geometry (the threshold displacement required to straighten the nonlinear spring) and is insensitive to the exact value of the $\mathrm{Si}_{3} \mathrm{~N}_{4}$ intrinsic film stress.

The inclined clamps also enable the tuning fork frequency to be insensitive to temperature changes. The working principle of temperature compensation is shown in Figure 1(e). When the temperature changes, the differential thermal expansion due to CTE mismatch between the $\mathrm{Si}_{3} \mathrm{~N}_{4}$ device layer and the silicon substrate results in thermal stress in the uncompensated devices. Specifically, the $\mathrm{Si}$ substrate expands more than the $\mathrm{Si}_{3} \mathrm{~N}_{4}$ device layer when the temperature increases, increasing the beam stress and in turn the resonant frequency of the tuning fork. However, in the compensated devices, the temperature-induced stress from the tension bar is balanced by that from the inclined clamps. Thus, the tuning fork beam anchor remains fixed regardless of temperature variation, providing a temperature independent mechanical frequency. The compensation strength can also be tuned by the clamp geometry. For example, the temperature induced force from the compensation clamps can be tuned by the width of the nonlinear springs. The temperature induced force from the tension bar can be tuned by the width of the tension bar. As a result, small, proper, and large ratios of nonlinear-spring to tension-bar width can produce undercompensated, properly compensated, and over-compensated tuning forks, respectively. FEM simulation shows that a device with a $3.2 \mu \mathrm{m}$ wide tension bar and $200 \mathrm{~nm}$ wide nonlinear springs can have almost zero temperature sensitivity. Thus, to investigate the influence of the width ratio between the nonlinear spring and the tension bar on the compensation strength, we fabricated devices with the nominal tension bar width varying from $2.8 \mu \mathrm{m}$ to $3.7 \mu \mathrm{m}$ and nonlinear spring widths varied from $194 \mathrm{~nm}$ to $211 \mathrm{~nm}$.

The mechanical frequencies of the tuning forks are measured through a near-field cavity-optomechanical readout [9]. Each tuning fork is near-field coupled across an $\approx 150 \mathrm{~nm}$ gap to a $\mathrm{Si}_{3} \mathrm{~N}_{4}$ microdisk optical cavity. The disk has a nominal diameter of $14 \mu \mathrm{m}$ and supports whispering-gallery (WGM) optical modes. When the light wavelength is tuned to the shoulder of a microdisk optical resonance, the tuning fork beam motion can modulate the optical resonance and hence change the transmitted light intensity. Then the output light signal can be transduced into electric signal by a photodetector for post processing. The optomechanical coupling parameter $g_{\mathrm{om}} / 2 \pi$ between the tuning fork in-plane squeezing mechanical mode and the microdisk WGM optical mode is calculated to be $\approx 140 \mathrm{MHz} / \mathrm{nm}$, which indicates the optical resonance frequency shift per unit modal displacement of the 
mechanical resonator. This near-field optical readout yields $\approx 1 \mathrm{fm} / \mathrm{Hz}^{0.5}$ displacement resolution [9] and enables a monolithic, robust, and fiber-pigtailed sensor technology [10].
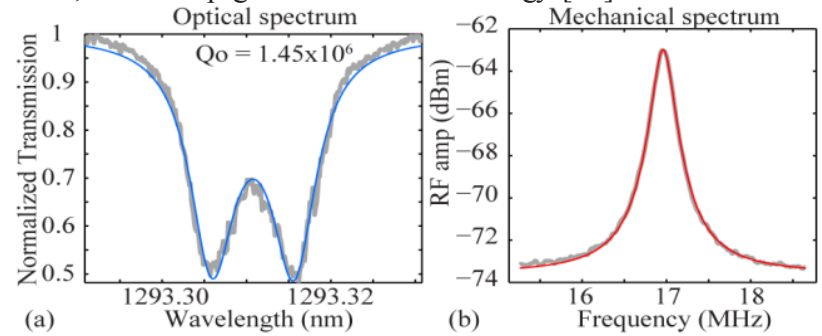

Figure 2. Experimentally measured (a) optical spectrum (b) mechanical spectrum. Colored lines are theory fits.

To measure the mechanical frequencies, light was emitted from a $1300 \mathrm{~nm}$ wavelength band tunable laser and evanescently coupled to the optical microdisk resonator through a fiber taperhelix probe [11]. The transmitted light intensity was measured by a photo detector (PD), with the output split into two channels. One channel connected to a data acquisition (DAQ) board for swept-wavelength spectroscopy of the optical cavity modes (Figure 2(a)). The other channel connected to an Electrical Spectrum Analyzer (ESA) to measure the mechanical frequency (Figure 2(b)). Since the optomechanical transduction noise is well below the random motion of the tuning fork driven by thermomechanical noise [12], the mechanical frequencies were measured from the thermal motion spectra of the tuning forks without external excitation. The temperature was stably controlled within $\pm 0.1 \mathrm{~K}$ by a ceramic heater with a proportional-integral-derivative (PID) controller. The device was enclosed inside an acrylic chamber, with desiccant boxes placed in the chamber and constant desiccated air flowing through the chamber, to minimize the influence from moisture on the devices. At room temperature the frequencies of the first-order squeezing mode were $\approx 27.7 \mathrm{MHz}$ and $\approx 16.5 \mathrm{MHz}$ for the uncompensated and compensated tuning forks, respectively.

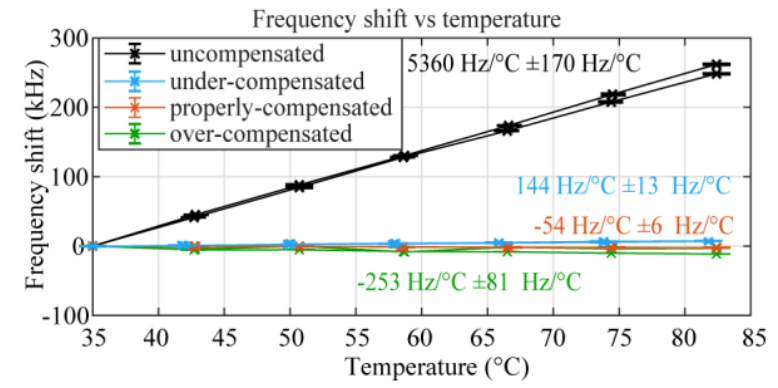

Figure 3. Experimentally measured temperature induced frequency shift. The frequency uncertainties are determined by nonlinear least squares fits to the data. The uncertainties for the temperature sensitivity are determined by the linear least squares fits.

To verify the temperature compensation effect, the mechanical frequency shifts of different devices over the temperature range from $35{ }^{\circ} \mathrm{C}$ to $82{ }^{\circ} \mathrm{C}$ were measured and shown in Figure 3. The resonant frequency of uncompensated device increased linearly with temperature at the rate of $\approx 5360 \mathrm{~Hz} / \mathrm{K} \pm 170 \mathrm{~Hz} / \mathrm{K}$. For a beam vibration dominated by the tensile stress, the fundamental mode resonant frequency $f_{\mathrm{m}}$ as a function of temperature $T$ can be described as [6]

$$
f_{m}(T)=\frac{1}{2 L} \sqrt{\frac{\sigma-A E\left(\alpha_{\text {beam }}-\alpha_{\text {sub }}\right)\left(T-T_{0}\right)}{\rho}}
$$

where $L$ is the beam length, $\sigma$ is the tensile stress along the beam at reference temperature, $\rho$ is the density of the beam, $E$ is the Young's modulus of the beam material, $\alpha_{\text {beam }}$ and $\alpha_{\text {sub }}$ are the CTE of the beam and substrate, respectively, and $T_{0}$ is the reference temperature. For the tuning fork with a tension bar, the stress is amplified by an amplification ratio $A=\sigma / \sigma_{0}$, and the stress variation due to temperature is similarly amplified. As a result, for the tuning fork with stress tuning, equation (1) is modified,

$$
f_{m}(T)=\frac{1}{2 L} \sqrt{\frac{\sigma-A E\left(\alpha_{\text {beam }}-\alpha_{\text {sub }}\right)\left(T-T_{0}\right)}{\rho}}
$$

in which $\sigma$ is the amplified beam stress. Since the CTE of the $\mathrm{Si}$ substrate $\left(\approx 2.6 \cdot 10^{-6} \mathrm{~K}^{-1}\right)$ is larger than that of $\mathrm{Si}_{3} \mathrm{~N}_{4}$ device layer $\left(\approx 1.6 \cdot 10^{-6} \mathrm{~K}^{-1}\right)$, the $f_{\mathrm{m}}$ increases as temperature increases. In addition, because $\sigma \approx 1.81 \mathrm{GPa}$ (calculated by FEM) is much larger than $E\left(\alpha_{\text {beam }}-\alpha_{\text {sub }}\right) T_{0}$, the higher order terms of $f_{\mathrm{m}}(T)$ can be neglected. Thus, $f_{\mathrm{m}}$ is approximately linearly-proportional to $T$ which agrees with the exp eriment result. The temperature sensitivity can be then calculated as

$$
S=\left.\frac{\partial f_{m}}{\partial T}\right|_{T=T_{0}}=-\frac{A E\left(\alpha_{\text {beam }}-\alpha_{s u b}\right)}{4 L \sqrt{\rho \sigma}}
$$

By using FEM-calculated values of $A \approx 3.12, E \approx 310 \mathrm{GPa}$ and $\rho \approx 3000 \mathrm{~kg} / \mathrm{m}^{3}$ for the $\mathrm{Si}_{3} \mathrm{~N}_{4}$ film, the temperature sensitivity is calculated to be $\approx 5200 \mathrm{~Hz} / \mathrm{K}$, which also matches the experiment.

For compensated devices, the resonant frequencies are also linearly-prop ortional to the temperature change but with much lower temperature sensitivity. This indicates that the nonlinear springs are fully deployed and the differential thermal strain is compensated by the nonlinear clamp design. The two inclined compensation clamps pull the center of the $\mathrm{Y}$ shaped structure in the direction opposite to that of the tension bar, balancing out the thermal stress induced motion. In addition, the strength of the compensation achieved by the Y-shaped clamp can be engineered by changing the width ratio between nonlinear springs and tension bar. With a proper width ratio, the temperature-induced movement of the center of Y-shaped clamp is such that the tuning fork does not experience a change in stress, which results in a perfect temperature compensation in the tuning fork. The thermal stress in the tension bar is dominant if the width ratio is too small, resulting in under-compensation, while a too large width ratio results in over-compensation.

As shown in Figure 3, three temperature compensation strengths are experimentally demonstrated using different nonlinear spring to tension-bar width ratios. Specifically, a device with nominally $177 \mathrm{~nm}$ wide nonlinear springs and a $3.14 \mu \mathrm{m}$ wide tension bar (width ratio 0.056 ) has a temperature sensitivity of $144 \mathrm{~Hz} / \mathrm{K} \pm 13 \mathrm{~Hz} / \mathrm{K}$, indicating this device is under-compensated. A device with $-253 \mathrm{~Hz} / \mathrm{K} \pm 81 \mathrm{~Hz} / \mathrm{K}$ temperature sensitivity (overcompensated) has $230 \mathrm{~nm}$ wide nonlinear springs and a $3 \mu \mathrm{m}$ wide tension bar (width ratio 0.077). The minimum temperature sensitivity $-54 \mathrm{~Hz} / \mathrm{K} \pm 6 \mathrm{~Hz} / \mathrm{K}$ (fractional frequency sensitivity of $\left.(3.2 \pm 0.4) \cdot 10^{-6} \mathrm{~K}^{-1}\right)$ is measured from device with a $183 \mathrm{~nm}$ wide spring and a $2.96 \mu \mathrm{m}$ wide tension bar (width ratio 0.062). Comparing to the uncompensated tuning fork which has $5360 \mathrm{~Hz} / \mathrm{K}$ $\pm 170 \mathrm{~Hz} / \mathrm{K}$ (fractional sensitivity $\left.(193 \pm 6) \cdot 10^{-6} \mathrm{~K}^{-1}\right)$, the temperature sensitivity is reduced by a factor of $\approx 60$ using the temperature compensation design. Temperature sensitivity uncertainties are determined from the linear fits.

Although our devices have not yet been tested directly as mechanical motion sensors, the displacement sensitivity of the resonance frequency is numerically and analytically estimated to be $\approx 51 \mathrm{kHz} / \mathrm{nm}$ for the uncompensated fork and $\approx 74 \mathrm{kHz} / \mathrm{nm}$ for the compensated fork based on the device dimension and resonant frequency measured in the experiments. These results confirm that the additional inclined clamps help to reduce the temperature induced frequency variability and the tuning fork transducer can be potentially used for force and displacement sensing with better stability. 

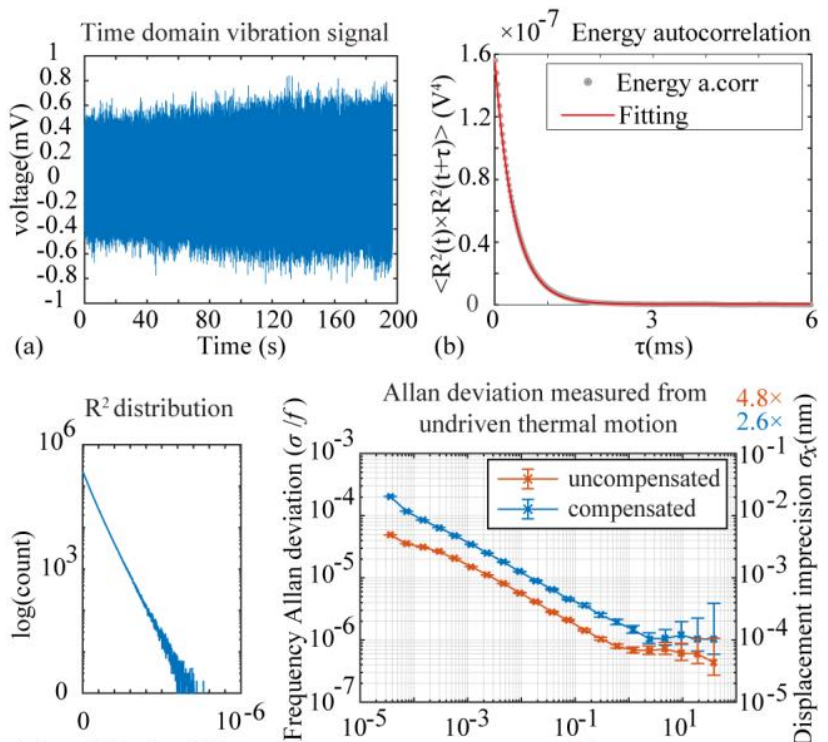

(c) $\mathrm{R}^{2}$ value $\left(\mathrm{V}^{2}\right)$

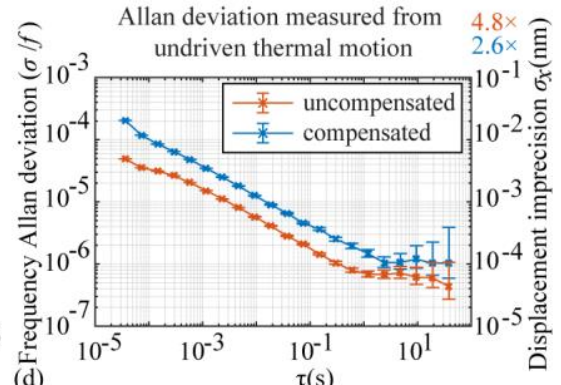

Figure 4. (a). Tuning fork time domain vibration signal. (b). Energy autocorrelation calculated from time domain signal. (c). $R^{2}$ distribution in log scale. (d). Experimentally measured frequency Allan deviation for compensated and uncompensated tuning forks, without excitation. Right-hand-side axis shows the corresponding calculated Allan deviation for a displacement sensing application with scale multipliers $4.8 \times$ (uncompensated) and $2.6 \times$ (compensated) due to different displacement sensitivity. The uncertainties for Allan deviation are determined by Chi distribution.

To further investigate the frequency stability of the tuning fork resonators, the time-domain vibration signal (quadrature, IQ, components at resonance) of the tuning fork thermal motion was also measured in vacuum using a lock-in amplifier. The I component of the uncompensated tuning fork time domain vibration signal is shown in Figure 4(a). Figure 4(b) shows the auto-correlation of its potential energy $\mathrm{Sa}(\tau)=<\mathrm{R}^{2}(\mathrm{t}) \times \mathrm{R}^{2}(\mathrm{t}+\tau)>$ where $\mathrm{R}$ is the signal amplitude. The calculated energy relaxation time $\mathrm{T}_{1} \approx 10^{-4} \mathrm{~s}$ indicates a sensor bandwidth exceeding $1 \mathrm{kHz}$. Figure 4(c) shows the distribution of $\mathrm{R}^{2}$, which is a straight line in log scale indicating that, as expected, the energy obeys the Maxwell-Boltzmann distribution $\left(\propto \exp \left(-\alpha \mathrm{R}^{2} /\left(2 k_{\mathrm{B}} \mathrm{T}\right)\right)\right)$, where $k_{\mathrm{B}}$ is the Boltzmann constant and $\alpha$ is a constant. This indicates the linearity of the readout and of the system itself being mechanically linear and in thermodynamic equilibrium. From the time domain data, the frequency was measured as a function of time and the Allan deviation calculated, shown in Figure 4(d). The Allan deviation slope measured for the uncompensated tuning fork with $28 \mathrm{MHz}$ mechanical frequency is $\approx 10^{-6} \mathrm{~Hz}^{-0.5}$ below $1 \mathrm{~s}$ averaging, which is thermodynamically limited [12]. The relative bias stability is $\approx 10^{-6}$ above $1 \mathrm{~s}$ averaging. The Allan deviation measured from the compensated device is similar to that for the uncompensated one, indicating that the temperature compensation is achieved without increasing noise from other noise sources [12].

In conclusion, we have developed $\mathrm{Si}_{3} \mathrm{~N}_{4}$ tuning fork cavity optomechanical transducers with nonlinear temperature compensation clamps. The clamp design allows the tuning fork mechanical frequency to be determined only by the geometry, regardless of the $\mathrm{Si}_{3} \mathrm{~N}_{4}$ intrinsic film stress. Control and reduction of temperature sensitivity was experimentally demonstrated by tuning the design geometry. Temperature sensitivity ranges from $\quad-253 \mathrm{~Hz} / \mathrm{K} \pm 81 \mathrm{~Hz} / \mathrm{K} \quad$ (over-compensation) to $144 \mathrm{~Hz} / \mathrm{K} \pm 13 \mathrm{~Hz} / \mathrm{K}$ (under-compensation) are achieved. The minimum temperature sensitivity is $-54 \mathrm{~Hz} / \mathrm{K} \pm 6 \mathrm{~Hz} / \mathrm{K}$ while that of an uncompensated tuning fork is $5360 \mathrm{~Hz} / \mathrm{K} \pm 170 \mathrm{~Hz} / \mathrm{K}$. The slope of measured thermodynamically-limited Allan deviation is $\approx 10^{-6} \mathrm{~Hz}^{-0.5}$ below $1 \mathrm{~s}$ averaging and the relative bias stability is $\approx 10^{-6}$ above $1 \mathrm{~s}$ averaging. The temperature-compensated resonant transducer presented here demonstrates a promising new highprecision, low-drift displacement measurement approach that can enable a variety of precision MEMS sensor applications.

\section{REFERENCES}

[1] K. Y. Yasumura, T. D. Stowe, E. M. Chow, T. Pfafman, T. W. Kenny, B. C. Stipe, et al., "Quality factors in micron-and submicron-thick cantilevers," Microelectromechanical Systems, Journal of, vol. 9, pp. 117-125, 2000.

[2] A. Suhel, B. D. Hauer, T. S. Biswas, K. S. D. Beach, and J. P. Davis, "Dissipation mechanisms in thermomechanically driven silicon nitride nanostrings," Applied Physics Letters, vol. 100, p. 173111, 2012.

[3] R. Zhang, C. Ti, M. I. Davanço, Y. Ren, V. Aksyuk, Y. Liu, et al., "Integrated tuning fork nanocavity optomechanical transducers with high $f_{\mathrm{M}} Q_{\mathrm{M}}$ product and stress-engineered frequency tuning," Applied Physics Letters, vol. 107, p. 131110, 2015.

[4] P. Temple-Boyer, C. Rossi, E. Saint-Etienne, and E. Scheid, "Residual stress in low pressure chemical vapor deposition SiNx films deposited from silane and ammonia," Journal of Vacuum Science \& Technology A: Vacuum, Surfaces, and Films, vol. 16, pp. 2003-2007, 1998.

[5] T. Kose, K. Azgin, and T. Akin, "Design and fabrication of a high performance resonant MEMS temperature sensor," Journal of Micromechanics and Microengineering, vol. 26, p. 045012, 2016.

[6] T. Larsen, S. Schmid, L. Grönberg, A. Niskanen, J. Hassel, S. Dohn, et al., "Ultrasensitive string-based temperature sensors," Applied Phy sics Letters, vol. 98, p. 121901, 2011.

[7] D. R. Myers, R. G. Azevedo, L. Chen, M. Mehregany, and A. P. Pisano, "Passive Substrate Temperature Compensation of Doubly Anchored Double-Ended Tuning Forks," Journal of Microelectromechanical Systems, vol. 21, pp. 1321-1328, 2012.

[8] J. C. Salvia, R. Melamud, S. A. Chandorkar, S. F. Lord, and T. W. Kenny, "Real-Time Temperature Compensation of MEMS Oscillators Using an Integrated Micro-Oven and a PhaseLocked Loop," Journal of Microelectromechanical Systems, vol. 19, pp. 192-201, 2010.

[9] Y. Liu, H. Miao, V. Aksyuk, and K. Srinivasan, "Wide cantilever stiffness range cavity optomechanical sensors for atomic force microscopy," Optics express, vol. 20, pp. 1826818280, 2012.

[10] J. Chae, S. An, G. Ramer, V. Stavila, G. Holland, Y. Yoon, et al., "Nanophotonic Atomic Force Microscope Transducers Enable Chemical Composition and Thermal Conductivity Measurements at the Nanoscale," Nano Letters, vol. 17, pp. 5587-5594, 2017/09/13 2017.

[11] Y. Ren, R. Zhang, C. Ti, and Y. Liu, "Tapered optical fiber loops and helices for integrated photonic device characterization and microfluidic roller coasters," Optica, vol. 3, pp. 1205-1208, 2016.

[12] M. Sansa, E. Sage, E. C. Bullard, M. Gély, T. Alava, E. Colinet, et al., "Frequency fluctuations in silicon nanoresonators," Nature Nanotechnology, vol. 11, p. 552, 02/29/online 2016.

\section{CONTACT}

*V. Aksyuk, tel:+1-301-975-2867; vladimir.aksyuk@nist.gov 